\title{
Exceptional odd-frequency pairing in non-Hermitian superconducting systems
}

\author{
Jorge Cayao and Annica M. Black-Schaffer \\ Department of Physics and Astronomy, Uppsala University, Box 516, S-751 20 Uppsala, Sweden
}

(Received 16 July 2021; revised 16 February 2022; accepted 24 February 2022; published 4 March 2022)

\begin{abstract}
We first show the realization of exceptional points in a non-Hermitian superconducting system based on a conventional superconductor and then demonstrate that, surprisingly, the system hosts odd-frequency pairing, solely generated by the non-Hermiticity. While there is a coexistence of even- and odd-frequency pairs under general conditions, we find that the even-frequency term vanishes at the exceptional degeneracies, leaving only odd-frequency pairing. This exceptional odd-frequency pairing is directly given by the imaginary part of the eigenvalues at the exceptional points and can be measured from the spectral function. Our results thus put forward non-Hermitian systems as a powerful platform to realize odd-frequency superconducting pairing.
\end{abstract}

DOI: 10.1103/PhysRevB.105.094502

\section{INTRODUCTION}

Superconductivity is a rare manifestation of quantum mechanics on a truly macroscopic scale and is also a basic ingredient in emerging quantum technologies [1]. To date, many superconducting states have been reported, both intrinsic and engineered using conventional $s$-wave superconductors in proximity to other materials, such as topological superconductivity in various hybrid devices [2,3]. While the scheme for creating unconventional superconductors may differ, their properties are always to a very large extent dictated by the symmetries of their fundamental constituents, the electron, or Cooper, pairs.

The Cooper pair wave function, or pair amplitude, depends on the degrees of freedom of the paired electrons [4]. While all the degrees of freedom are important for the Cooper pair symmetries, it is perhaps the time at which electrons pair that introduces the most interesting but least explored properties, mainly due to their relevance in dynamic quantum matter [5]. In its most general form, electrons can pair at different times, or equivalently at finite frequency $\omega$. This enables odd-frequency (odd- $\omega$ ) pairing, where the pair amplitude is odd in relative time, or equivalently odd in $\omega$. Odd- $\omega$ pairing is thus an intrinsically dynamic and time-dependent effect [6-10].

Since its initial conception [11], odd- $\omega$ pairing has generated an ever increasing interest, not only due to its dynamical nature but also because it explains several exotic effects, such as long-range proximity effects or paramagnetic Meissner signatures [6-10]. Interestingly, odd- $\omega$ pairs have been shown to emerge in several systems using just conventional $s$-wave

Published by the American Physical Society under the terms of the Creative Commons Attribution 4.0 International license. Further distribution of this work must maintain attribution to the author(s) and the published article's title, journal citation, and DOI. Funded by Bibsam. superconductors, with notable examples in superconducting heterostructures [12-18], multiband superconductors [19-24], and time-periodic superconductors [25,26]. Still, these systems share a common characteristic in that all represent closed systems, described by Hermitian Hamiltonians.

Physical systems are, however, always coupled to their environment, and thus open, where dissipative effects are unavoidable and described by non-Hermitian (NH) processes [27]. Notably, dissipation has been shown to lead to unique $\mathrm{NH}$ effects that broaden the system symmetries [28], giving rise to unusual phases [29-31] with no analog in Hermitian setups. The main property of NH systems is that they exhibit a complex spectrum with level degeneracies, known as exceptional points (EPs) [32-40], where eigenstates and eigenvalues coalesce, in stark contrast to Hermitian systems. Moreover, non-Hermiticity not only allows to understand and engineer dissipative systems, but it can also be precisely controlled and hence used for sophisticated applications [29-31], such as for high-performance lasers [41-44] and sensors [45-48].

Non-Hermiticity has also recently been shown to ramify the particle-hole symmetry [28], intrinsic in superconductors. It is thus natural to ask about its impact on the symmetry of the pair amplitude. Moreover, due to the close link between non-Hermiticity and dissipation, which reflects a dynamical essence, it represents a genuinely promising ground to explore as the origin of odd- $\omega$ pairing. However, the connection between non-Hermiticity and odd- $\omega$ pairing has so far received little attention, with studies only focusing on symmetry classification [49] or spectral broadening in a Dynes superconductor [50]. This has left, for example, the role of the main $\mathrm{NH}$ characteristic, the EPs, completely unexplored.

In this paper we first show how $\mathrm{NH}$ superconducting systems easily host odd- $\omega$ pairing, entirely due to nonHermiticity. Surprisingly, we find that all even- $\omega$ pairing vanishes at the EPs, leaving only a large odd- $\omega$ contribution, which we refer to as exceptional odd- $\omega$ pairing. We then illustrate these results in a realistic NH system consisting of a conventional superconductor coupled to a ferromagnet lead 


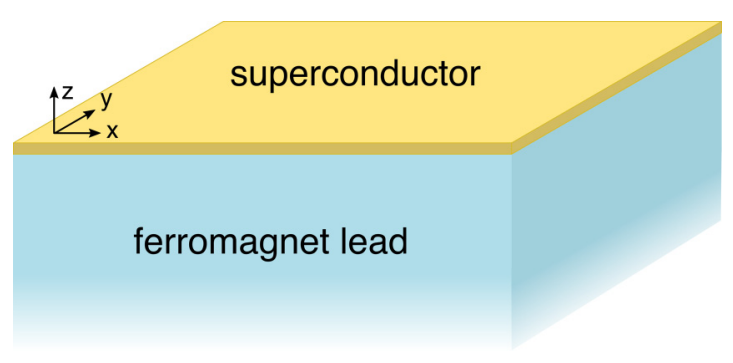

FIG. 1. Sketch of a two-dimensional (2D) conventional $s$-wave superconductor coupled to a semi-infinite ferromagnet lead. Due to coupling to the lead, the total system is described by an effective non-Hermitian Hamiltonian.

(see Fig. 1). Finally, we show that the exceptional odd- $\omega$ pairing, as well as the EPs, can be detected in the spectral function via angle-resolved photoemission spectroscopy (ARPES). Our findings thus put forward an entirely different route for generating odd- $\omega$ pairing, paving the way for $\mathrm{NH}$ engineering of dynamical superconducting states.

\section{PAIR AMPLITUDES}

To understand how odd- $\omega$ pairing appears in NH systems, we first inspect the structure of the pair amplitude $F$, which is obtained from the electron-hole (eh), or anomalous, part of the Green's function $G(\omega)=(\omega-H)^{-1}$ [51,52]. Here, $H$ is the system Hamiltonian in Nambu space $\psi=\left(c, c^{\dagger}\right)^{\mathrm{T}}$, where $c$ annihilates an electronic state. While $F$ can be directly found from a matrix inversion, to gain a basic understanding of its dependencies it is more useful to express $(\omega-H)^{-1}$ in terms of its adjugate (Adj) and determinant (det) [53]. In this way, $F$ reads

$$
F(\omega)=\frac{1}{\operatorname{det}(\omega-H)}[\operatorname{Adj}(\omega-H)]_{\mathrm{eh}},
$$

with $\operatorname{Adj}(\cdot)$ found as the transpose of the cofactor matrix [53]. The representation of $F$ in Eq. (1) is general and valid for both Hermitian and NH Hamiltonians.

While Eq. (1) might seem complicated, it actually offers a simple way to analyze how odd- $\omega$ pairing appears, as any odd- $\omega$ part must come either from the denominator or numerator. For this reason, we first note that the poles of $G$ give the quasiparticle energies, or the eigenvalues $E_{i}$ of $H$. Then, to visualize the appearance of odd- $\omega$ pairing in Eq. (1) it is convenient to express the determinant in terms of $E_{i}$ : $\operatorname{det}(\omega-H)=\Pi_{i}\left(\omega-E_{i}\right)$ [53]. For simplicity, but without loss of generality, we for now assume that spin, space, and orbital are not active degrees of freedom, such that $H$ only has two eigenvalues $E_{1,2}$. Thus, we can write $\operatorname{det}(\omega-H)=$ $\left(\omega-E_{1}\right)\left(\omega-E_{2}\right)$, with $E_{1,2}$ related by particle-hole symmetry, which can differ for Hermitian and NH Hamiltonians [28].

For Hermitian systems, $E_{1,2}= \pm E$ and the denominator of Eq. (1) becomes $\operatorname{det}(\omega-H)=\omega^{2}-E^{2}$, clearly an even function of $\omega$. Also, the numerator of Eq. (1), $\operatorname{Adj}(\cdot)$, does not develop any odd- $\omega$ term in this simple case. However, we have verified that in systems with finite odd- $\omega$ pairing, such as two-band superconductors [10], it is the $[\operatorname{Adj}(\cdot)]_{\text {eh }}$ term that generates odd- $\omega$ pairing, while $\operatorname{det}(\cdot)$ only provides even powers of $\omega$. Thus, for time-independent Hermitian Hamiltonians with the properties discussed above, the only option for $F$ to contain odd- $\omega$ pairing comes from the $[\operatorname{Adj}(\cdot)]_{\text {eh }}$ matrix.

In contrast, for $\mathrm{NH}$ systems the eigenvalues are no longer real (Re) but develop an imaginary (Im) term, $E_{n}=a_{n}-i b_{n}$, with $a, b$ both real-valued numbers [54]. For NH superconducting systems, they come in pairs, obeying $E_{1}=-E_{2}^{*}$ due to the charge-conjugation symmetry [28,55-58]. This imposes $a_{1}=-a_{2}=a$ and $b_{1}=b_{2} \equiv b$. Then, the denominator in Eq. (1) reads $\operatorname{det}(\omega-H)=\omega^{2}-a^{2}-b^{2}+2 i \omega b$, where the last term now directly reveals an odd- $\omega$ term proportional to $b$, while the numerator of Eq. (1) still does not contain any odd- $\omega$ part. Taken together, the pair amplitude of NH systems reads

$$
F_{\mathrm{NH}}(\omega)=\frac{[\operatorname{Adj}(\omega-H)]_{\mathrm{eh}}}{d^{2}+4 \omega^{2} b^{2}}(d-2 i \omega b),
$$

where $d=\omega^{2}-a^{2}-b^{2}$ is an even function of $\omega$. This $F_{\mathrm{NH}}$ has both even- and odd- $\omega$ parts, proportional to $d$ and $i \omega b$, respectively. Importantly, the odd- $\omega$ term is purely driven by the Im part of the eigenvalues, $b$.

The main characteristic of NH Hamiltonians is the presence of EPs, where eigenvalues and eigenvectors coalesce [32-35]. This implies that at the EPs, $a_{1}=-a_{2}=0$ and $b_{1}=b_{2}=b$, leaving a single purely Im eigenvalue, $E_{1,2}=i b$. Also, then $d=\omega^{2}-b^{2}$, which vanishes when $\omega=|b|$, i.e., at the EP. Hence, at the EP, the even- $\omega$ term of $F_{\mathrm{NH}}$ vanishes, leaving only odd- $\omega$ pairing, which we refer to as exceptional odd- $\omega$ pairing. We thus conclude that odd- $\omega$ pairing can be easily induced in a $\mathrm{NH}$ system, even when it is completely absent in the Hermitian regime, and even more interestingly, it becomes the only source of pairing at EPs.

\section{REALIZATION OF A NH SUPERCONDUCTING SYSTEM}

Next, we show that odd- $\omega$ pairing emerges naturally in realistic NH systems. For this purpose, we first engineer a simple NH superconducting system by coupling a conventional spin-singlet $s$-wave 2D superconductor [59-67] to a ferromagnetic lead (see Fig. 1). This NH system is modeled by the following effective Nambu Hamiltonian,

$$
H_{\text {eff }}=H_{\mathrm{S}}+\Sigma^{r}(\omega=0),
$$

where $H_{\mathrm{S}}=\xi_{k} \tau_{z}-\Delta \sigma_{y} \tau_{y}$ describes the (closed) superconductor in the basis $\left(c_{k, \uparrow}, c_{k, \downarrow}, c_{-k, \uparrow}^{\dagger}, c_{-k, \downarrow}^{\dagger}\right)$, with $c_{k, \sigma}$ annihilating an electron with momentum $k$ and spin $\sigma$. Here, $\xi_{k}=\hbar^{2} k^{2} / 2 m-\mu$ is the kinetic energy with $k=\left(k_{x}, k_{y}\right)$, $\sigma_{i}$ and $\tau_{i}$ the spin and electron-hole Pauli matrices, respectively, $\mu$ is the chemical potential, and $\Delta$ is the spin-singlet $s$-wave pair potential. We consider either intrinsic thin film superconductors or proximity-induced superconductivity into a thin film semiconductor, both effectively producing a $2 \mathrm{D}$ superconductor, but our results are also valid in the interface region for 3D superconductors [68]. Further, $\Sigma^{r}(\omega=0)$ is the retarded spin-dependent self-energy at $\omega=0$ describing the effect of the lead on the superconductor. While $\Sigma^{r}$, in general, depends on $\omega$, its independence of $\omega$ is well justified e.g., in the wideband limit [69-72]. With the lead being semi-infinite, $\Sigma^{r}$ has both Re and Im terms. While the Re part is Hermitian and just renormalizes the elements of $H_{\mathrm{S}}$, the Im part is $\mathrm{NH}$ 


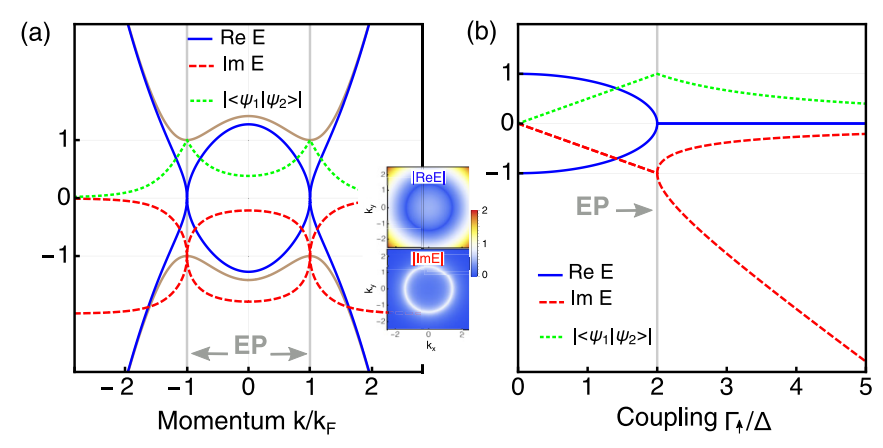

FIG. 2. Re (blue) and Im (red) parts of the eigenvalues in Eq. (5) as a function of $k$ at fixed $\Gamma_{\uparrow, \downarrow}$ (a) and as a function of $\Gamma_{\uparrow}$ at fixed $\xi_{k}=0$ and $\Gamma_{\downarrow}=0$ (b), with the wave-function overlap in green. At the EP transition (gray) the eigenvalues coalesce and the wave functions become parallel. The brown curve shows eigenvalues without non-Hermiticity. The inset depicts the absolute value of Re and Im parts of the eigenvalues. Parameters: $\Gamma_{\uparrow}=2, \Gamma_{\downarrow}=0, \Delta=1$, $\mu=1, k_{\mathrm{F}}=\sqrt{2 m \mu / \hbar^{2}}$.

and introduces dramatic changes, which becomes our focus here $[69,71,72]$. We obtain $\Sigma^{r}(\omega=0)=\operatorname{diag}\left(\Sigma_{\mathrm{e}}^{r}, \Sigma_{\mathrm{h}}^{r}\right)$ analytically [see Supplemental Material (SM) for details [73]], where we approximate [74]

$$
\Sigma_{\mathrm{e}, \mathrm{h}}^{r}(\omega=0)=-i \Gamma \sigma_{0}-i \gamma \sigma_{z},
$$

with $\Gamma=\left(\Gamma_{\uparrow}+\Gamma_{\downarrow}\right) / 2$ and $\gamma=\left(\Gamma_{\uparrow}-\Gamma_{\downarrow}\right) / 2$. Here, $\Gamma_{\sigma}=$ $\pi\left|t^{\prime}\right|^{2} \rho_{\mathrm{L}}^{\sigma}$ with $\rho_{\mathrm{L}}^{\sigma}$ the surface density of states of the lead (L) for spin $\sigma=\uparrow, \downarrow$, controlled by the Zeeman field in the ferromagnet, and $t^{\prime}$ the hopping amplitude into the lead from the superconductor. For obvious reasons we refer to $\Gamma_{i}$ as to the coupling amplitude. Due to causality, all terms in $\Sigma^{r}$ reside in the lower complex energy half plane, a clear signal of dissipation.

Using Eq. (4), the eigenvalues of $H_{\text {eff }}$ are given by

$$
E_{n}=-i \Gamma \pm \sqrt{\Delta^{2}+\xi_{k}^{2}-\gamma^{2} \pm 2 i\left|\xi_{k}\right||\gamma|},
$$

which acquire Im terms solely due to the effect of the lead through $\Gamma$ and $\gamma$. At $\Gamma=\gamma=0$, the system is Hermitian with real eigenvalues $E_{n}= \pm \sqrt{\Delta^{2}+\xi_{k}^{2}}$, shown in brown in Fig. 2(a). At any nonzero coupling, $E_{n}$ develops nonzero Im terms, a clear feature of $\mathrm{NH}$ physics. The inverse of $\operatorname{Im}\left(E_{n}\right)$ represents the average time a quasiparticle remains in the superconductor before escaping into the lead, setting the length scale $\ell_{\Gamma}=\hbar v_{\mathrm{F}} / \operatorname{Im}\left(E_{n}\right)$, with $v_{\mathrm{F}}$ the Fermi velocity in the superconductor, for how deep the NH effect penetrates if using a 3D superconductor. At $\Gamma_{\uparrow}=\Gamma_{\downarrow}, \gamma=0$ and all $E_{n}$ 's acquire the same Im term, equal to $-i \Gamma$. It is only when $\Gamma_{\uparrow} \neq \Gamma_{\downarrow}$ that all $E_{n}$ 's undergo the special transition at which their Re and Im parts merge into a single value, $i \Gamma$, thus producing EPs. This occurs when the square root in Eq. (5) vanishes,

$$
\Delta^{2}+\xi_{k}^{2}-\gamma^{2}=0 \text { and } 2 i\left|\xi_{k}\right||\gamma|=0 .
$$

To visualize these EP conditions, we present in Figs. 2(a) and 2(b) the Re (solid blue) and Im (dashed red) parts of $E_{n}$ as a function of $k$ and $\Gamma_{\uparrow}$, with the EP transitions marked in gray. We observe that the electron- and holelike $E_{n}$ coalesce, and EPs appear, only at $\xi_{k}=0$, or equivalently $k=\sqrt{2 m \mu / \hbar^{2}}$, provided $\Delta=|\gamma| \neq 0$. The EPs extend into a circle when $k$ is plotted in 2D (see the inset in Fig. 2). As expected for EPs, the conditions in Eqs. (6) not only define the coalescence of $E_{n}$, but they also define the coalescence of the associated eigenvectors. In fact, at the EPs, the associated wave vectors become parallel instead of orthogonal as for Hermitian systems, as seen by their scalar product (dotted green) in Fig. 2. In Fig. 2(b), we instead fix $\xi_{k}=0$ and plot the eigenvalues as a function of $\Gamma_{\uparrow}$ at fixed $\Gamma_{\downarrow}=0$ and again see a clear EP transition. Thus, our simple, but physical, NH superconducting system in Fig. 1 host clear and stable EPs, which represent the main property of $\mathrm{NH}$ systems $[30,31]$.

\section{EXCEPTIONAL ODD- $\omega$ PAIR AMPLITUDE}

Having established the existence of EPs in the NH system in Fig. 1 and Eq. (3), we next turn to calculating its pair amplitudes using the anomalous components of the retarded Green's function $G^{r}=\left(\omega-H_{\text {eff }}\right)^{-1}$. We obtain even- and odd- $\omega(\mathrm{E}, \mathrm{O})$ pair amplitudes given by

$$
F_{\uparrow \downarrow}^{\mathrm{E}}(\omega)=\frac{-\Delta Q_{\uparrow \downarrow}}{Q_{\uparrow \downarrow}^{2}+4 \omega^{2} \Gamma^{2}}, \quad F_{\uparrow \downarrow}^{\mathrm{O}}(\omega)=\frac{-2 i \omega \Delta \Gamma}{Q_{\uparrow \downarrow}^{2}+4 \omega^{2} \Gamma^{2}},
$$

where $Q_{\uparrow \downarrow}=\Delta^{2}+\xi_{k}^{2}+\Gamma^{2}-\gamma^{2}-\omega^{2}-2 i \gamma \xi_{k}$ is an even function in $\omega$. Likewise, we get $F_{\downarrow \uparrow}^{\mathrm{E}(\mathrm{O})}=-F_{\uparrow \downarrow}^{\mathrm{E}(\mathrm{O})}\left(\Gamma_{\uparrow} \leftrightarrow \Gamma_{\downarrow}\right)$, but we do not find any equal spin pairing. An interesting feature is that $F_{\uparrow \downarrow}^{\mathrm{O}}$ is proportional to $\Gamma$, showing that it is a direct $\mathrm{NH}$ result, as in Eq. (2). The finite pair amplitudes can also be interpreted as a result of Andreev reflection at the superconductor-lead interface [17,75-77].

To further inspect the $\mathrm{NH}$ effect on $F_{\downarrow \uparrow}^{\mathrm{E}, \mathrm{O}}$, we plot their absolute values in Fig. 3 as a function of $\omega, \Gamma_{\uparrow}$, and $k$. At $\Gamma=\gamma=0$, the system is Hermitian and then only the even- $\omega$ part survives, as seen both in Eqs. (7) and Fig. 3. At finite coupling, the system becomes $\mathrm{NH}$ and even- and odd- $\omega$ pairs generally coexist. As seen in Fig. 3, both pair amplitudes develop large values, but in different regimes, allowing us to establish a clear distinction between them: While $F_{\uparrow \downarrow}^{\mathrm{E}}$ is large around $\omega=0, F_{\uparrow \downarrow}^{\mathrm{O}}$ exhibits surprisingly similarly large values at higher $\omega[78]$.

Next, we examine the effect of EPs on $F_{\uparrow \downarrow}^{\mathrm{O}, \mathrm{E}}$ in Eqs. (7). For this reason we analyze the term $Q_{\uparrow \downarrow}$ at the EPs, where the latter are defined by the conditions in Eqs. (6) and only present for $\gamma \neq 0$. By using these EP conditions, we get $Q_{\uparrow \downarrow}=\Gamma^{2}-$ $\omega^{2}$, assuming we already have tuned $\Delta=|\gamma|$. Interestingly, $Q_{\uparrow \downarrow}$ vanishes exactly at $\omega=|\Gamma|$, i.e., exactly at the magnitude of the eigenvalues at EPs [see Eqs. (5)]. Thus, at the EPs, we find only odd- $\omega$ pairing $F_{\uparrow \downarrow}^{\mathrm{O}}(\omega)=-(i \Delta) /(2 \omega \Gamma)$, with $|\omega|=$ $\Gamma$, as the even- $\omega$ part identically vanishes. This exceptional odd- $\omega$ pairing is unusual for two additional reasons: Its size is solely determined by the $\mathrm{NH}$ processes $\Gamma$ and $\gamma$, as $|\omega|=\Gamma$ and $\Delta=|\gamma|$ at the EPs, and it has a clear $\operatorname{sgn}(\omega) / \omega^{2}$ behavior, unlike Hermitian systems [79]. In Fig. 3(a), the vanishing of the even- $\omega$ pairing actually occurs along the whole line $\omega=\Delta$ as $\Gamma_{\uparrow}$ is varied (white dashed line), although the EP only occurs at the point $\Gamma_{\uparrow} / \Delta=2$ and at $\omega / \Delta=1$ in this plot (green arrow). This is because the particular choice of parameters in Fig. 3(a) results in $Q_{\uparrow \downarrow}=0$ and thus zero even- $\omega$ pairing for all $|\omega|=\Delta$; note that the second condition for EPs, $\xi_{k}=0$, 

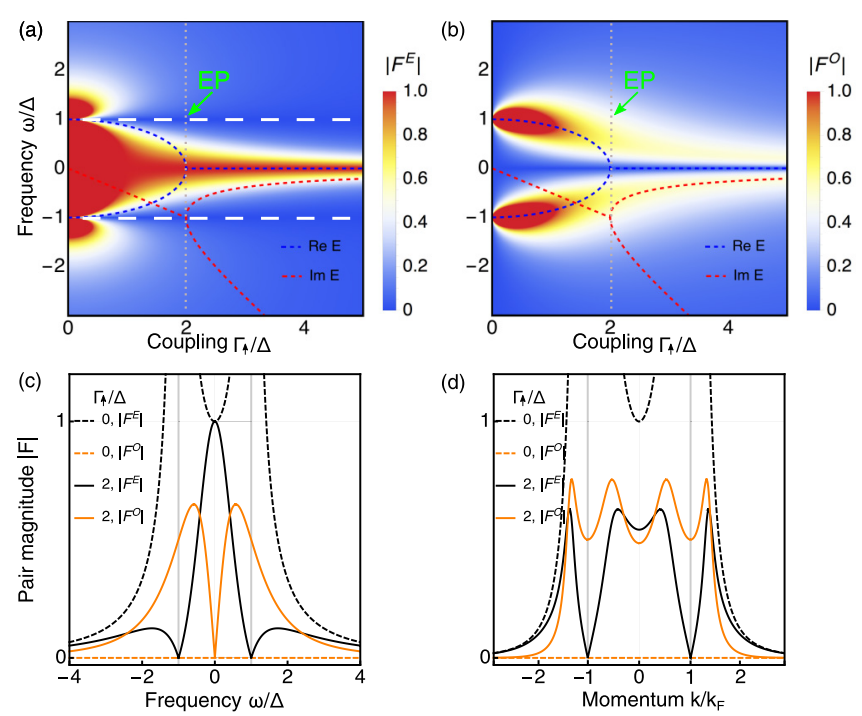

(d)

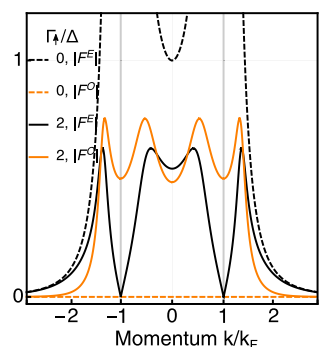

FIG. 3. Absolute value of (a) even- $\omega$ and (b) odd- $\omega$ pair amplitudes as a function of $\omega$ and $\Gamma_{\uparrow}$ at $\xi_{k}=0, \Gamma_{\downarrow}=0$, with the color scale cut off at 1 for visualization. Dashed blue and red curves show the Re and Im parts of the eigenvalues, respectively. Also marked are the EP transition lines (gray) and energy values (green arrows), and values where the even- $\omega$ pairing vanish (dashed white). Pair amplitudes (c) as a function of $\omega$ at $\xi_{k}=0$ and (d) as a function of $k$ at fixed $\omega / \Delta=1$ for different values of $\Gamma_{\uparrow}$. The rest of the parameters are as in Fig. 2.

in Eqs. (6), is satisfied here. We thus find that vanishing even- $\omega$ pairing is intimately related to the occurrence of EPs in our system, leaving only finite exceptional odd- $\omega$ pairing, which, in turn, is solely determined by the magnitude of the eigenvalues at the EPs.

\section{SPECTRAL SIGNATURES}

To detect the EPs and the odd- $\omega$ pairing, we study the spectral function $A(\omega, k)=-\operatorname{Im} \operatorname{Tr}\left(G^{\mathrm{r}}-G^{\mathrm{a}}\right)$ [51,52] accessible via, e.g., ARPES measurements [80-82], where $G^{\mathrm{a}}=\left[G^{\mathrm{r}}\right]^{\dagger}$ is the advanced Green's function [83]. To elucidate the pair amplitude dependency, it is useful to write the diagonal entries of $G^{\mathrm{r}}$ in terms of the pair amplitudes. The diagonal electron terms are thus given by

$$
\left[G_{0}^{r}(\omega)\right]_{\uparrow \uparrow(\downarrow \downarrow)}= \pm \frac{\left(\omega+\xi_{k}+i \Gamma_{\downarrow(\uparrow)}\right)}{\Delta}[F(\omega)]_{\uparrow \downarrow(\downarrow \uparrow)},
$$

with $F_{\uparrow \downarrow}=F_{\uparrow \downarrow}^{\mathrm{E}}+F_{\uparrow \downarrow}^{\mathrm{O}}$ given by Eqs. (7). The diagonal hole terms are $\left[\bar{G}_{0}^{r}\right]_{\uparrow \uparrow(\downarrow \downarrow)}=\left[G_{0}^{r}\right]_{\downarrow \downarrow(\uparrow \uparrow)}\left(\xi_{k} \rightarrow-\xi_{k}, \Gamma_{\uparrow(\downarrow)} \rightarrow\right.$ $\left.\Gamma_{\downarrow(\uparrow)}\right)$. We further isolate the individual even- and odd- $\omega$ pair contributions by writing $A=A^{\mathrm{E}}+A^{\mathrm{O}}$ with $A^{\mathrm{E}(\mathrm{O})}$ being due to $F_{a b}^{\mathrm{E}(\mathrm{O})}$.

In Fig. 4 we plot $A^{\mathrm{E}, \mathrm{O}}$ and $A$ as functions of $\omega$ and $\Gamma_{\uparrow}$ at $\xi_{k}=0$. By examining the individual contributions in Fig. 4(a), we note that they exhibit large values in different ranges of $\omega$ and $\Gamma_{\uparrow}$. In fact, $A^{\mathrm{E}}$ acquires large values around $\omega=0$ and high $\Gamma_{\uparrow}$, similar to $F_{\uparrow \downarrow}^{\mathrm{E}}$ in Fig. 3(a). Surprisingly, it also becomes negative for some parameters. On the other hand, $A^{\mathrm{O}}$ instead shows large values at finite $\omega$ and low $\Gamma_{\uparrow}$, stemming from large $F_{\uparrow \downarrow}^{\mathrm{O}}$ for the same parameters [see Fig. 3(b)].
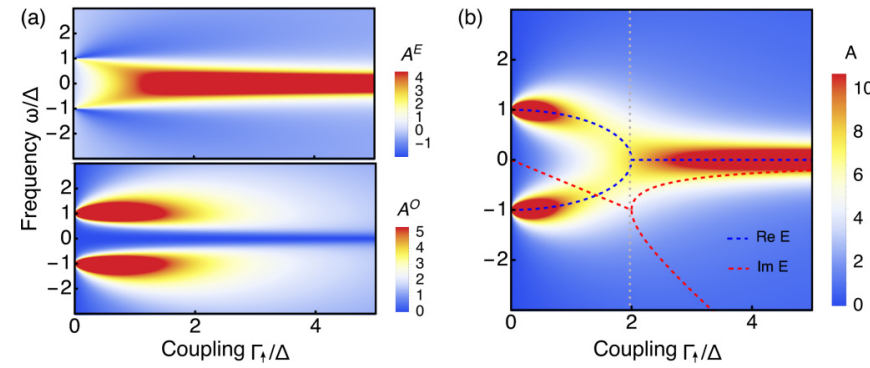

FIG. 4. (a) Spectral function $A$ divided into individual contributions $A^{E}$ from even- $\omega$ (top) and $A^{O}$ from odd- $\omega$ (bottom) pairing as a function of $\omega$ and $\Gamma_{\uparrow}$ at $\xi_{k}=0, \Gamma_{\downarrow}=0$. (b) Total spectral function $A=A^{\mathrm{E}}+A^{\mathrm{O}}$, with EP transition lines (gray) and Re (dashed blue) and $\mathrm{Im}$ (dashed red) parts of eigenvalues depicted. The rest of the parameters are as in Figs. 2 and 3.

The total spectral function $A$ in Fig. 4(b) captures the main features of both $A^{\mathrm{E}}$ and $A^{\mathrm{O}}$, where $A^{\mathrm{O}}$ also compensates for the negative values of $A^{\mathrm{E}}$. Note that $A$ also clearly signals the EP transition (gray line). In fact, at the EP energy, $|\omega|=\Gamma$, we estimate $A=A^{\mathrm{O}} \approx 2 \omega F^{\mathrm{O}} / \Delta$, with $F^{\mathrm{O}}=\Delta /(\omega \Gamma)$ being the magnitude of the exceptional odd- $\omega$ pairing. Thus, the spectral function detects the EP transition which then allows us to measure the exceptional odd- $\omega$ pairing.

Experimentally, to generate exceptional odd- $\omega$ pairing, high control of $\Delta$ and $\Gamma_{\sigma}$ is necessary. For $\Delta$, recent works have reported well-controlled proximity-induced superconductivity in only $a=7 \mathrm{~nm}$ thick InAs films with $\Delta=$ $0.2 \mathrm{meV}$ and tunable using interface barriers [59]. For $\Gamma_{\sigma}$, both the spin-dependent density of states and the tunneling between lead and superconductor can be tuned [see Eq. (4)]. Here, the Zeeman field of the lead guarantees distinct $\Gamma_{\sigma}$, while the overall strength can be controlled by adjusting the thickness of a normal potential barrier between superconductor and lead, e.g., by using a few nm thick InGaAs layer [59]. Along these lines, we estimate that Zeeman fields of $B=1 \mathrm{meV}$ produce couplings of $\Gamma_{\uparrow}=0.4 \mathrm{meV}$ and $\Gamma_{\downarrow}=0$, giving rise to $\gamma=\Delta$ and a length scale of $\ell_{\Gamma} \approx 120 \mathrm{~nm}$ (see SM [73]). Thus, currently available heterostructures achieve both the necessary EP conditions and exhibit $a \ll \xi_{\Gamma}$, assuring that exceptional odd- $\omega$ pairs can homogeneously emerge in such systems.

\section{CONCLUSIONS}

In conclusion, we have shown the emergence of EPs in simple and physical NH superconducting systems based on conventional superconductors. We have then demonstrated that such systems host odd- $\omega$ pairing purely due to the nonHermiticity, which, at the EPs becomes the only source of superconducting pairing, establishing the concept of exceptional odd- $\omega$ pairing. Finally, we showed how the spectral function can be used to detect both the emergence of EPs and measure exceptional odd- $\omega$ pairing. Our work puts forward $\mathrm{NH}$ systems as a rich playground for generating odd- $\omega$ pairs, paving the way for $\mathrm{NH}$ engineering of dynamical superconducting states with enhanced and controlled properties. 


\section{ACKNOWLEDGMENTS}

We thank E. J. Bergholtz, J. C. Budich, D. Chakraborty, and L. Potenciano and for insightful discussions. We also acknowledge financial support from the Swedish Research Council (Vetenskapsrådet Grants No. 2018-03488 and No. 2021-04121) and the European Research Council (ERC) under the European Unions Horizon 2020 research and innovation programme (ERC-2017-StG-757553).

[1] A. Acín, I. Bloch, H. Buhrman, T. Calarco, C. Eichler, J. Eisert, D. Esteve, N. Gisin, S. J. Glaser, F. Jelezko, S. Kuhr, M. Lewenstein, M. F. Riedel, P. O. Schmidt, R. Thew, A. Wallraff, I. Walmsley, and F. K. Wilhelm, The quantum technologies roadmap: A European community view, New J. Phys. 20, 080201 (2018).

[2] S. M. Frolov, M. J. Manfra, and J. D. Sau, Topological superconductivity in hybrid devices, Nat. Phys. 16, 718 (2020).

[3] K. Flensberg, F. von Oppen, and A. Stern, Engineered platforms for topological superconductivity and Majorana zero modes, Nat. Rev. Mater. 6, 944 (2021).

[4] M. Tinkham, Introduction to Superconductivity (Courier Corporation, Noth Chelmsford, MA, 2004).

[5] A. V. Balatsky, J. T. Haraldsen, A. M. Black-Schaffer, A. Pertsova, and P. Hofmann, Dynamic quantum matter, Ann. Phys. 532, 2000037 (2020).

[6] F. S. Bergeret, A. F. Volkov, and K. B. Efetov, Odd triplet superconductivity and related phenomena in superconductorferromagnet structures, Rev. Mod. Phys. 77, 1321 (2005).

[7] Y. Tanaka, M. Sato, and N. Nagaosa, Symmetry and topology in superconductors-odd-frequency pairing and edge states, J. Phys. Soc. Jpn. 81, 011013 (2012).

[8] J. Linder and A. V. Balatsky, Odd-frequency superconductivity, Rev. Mod. Phys. 91, 045005 (2019).

[9] J. Cayao, C. Triola, and A. M. Black-Schaffer, Odd-frequency superconducting pairing in one-dimensional systems, Eur. Phys. J. Spec. Top. 229, 545 (2020).

[10] C. Triola, J. Cayao, and A. M. Black-Schaffer, The role of oddfrequency pairing in multiband superconductors, Ann. Phys. 532, 1900298 (2020).

[11] V. L. Berezinskii, New model of the anisotropic phase of superfluid ${ }^{3} \mathrm{He}$, Zh. Eksp. Teor. Fiz. 20, 628 (1974) [JETP Lett. 20, 287 (1974)].

[12] F. S. Bergeret, A. F. Volkov, and K. B. Efetov, Long-Range Proximity Effects in Superconductor-Ferromagnet Structures, Phys. Rev. Lett. 86, 4096 (2001).

[13] A. Kadigrobov, R. I. Shekhter, and M. Jonson, Quantum spin fluctuations as a source of long-range proximity effects in diffusive ferromagnet-superconductor structures, Europhys. Lett. 54, 394 (2001).

[14] Y. Tanaka, Y. Tanuma, and A. A. Golubov, Odd-frequency pairing in normal-metal/superconductor junctions, Phys. Rev. B 76, 054522 (2007).

[15] A. M. Black-Schaffer and A. V. Balatsky, Proximity-induced unconventional superconductivity in topological insulators, Phys. Rev. B 87, 220506(R) (2013).

[16] F. Crépin, P. Burset, and B. Trauzettel, Odd-frequency triplet superconductivity at the helical edge of a topological insulator, Phys. Rev. B 92, 100507(R) (2015).
[17] J. Cayao and A. M. Black-Schaffer, Odd-frequency superconducting pairing and subgap density of states at the edge of a two-dimensional topological insulator without magnetism, Phys. Rev. B 96, 155426 (2017).

[18] C. Fleckenstein, N. T. Ziani, and B. Trauzettel, Conductance signatures of odd-frequency superconductivity in quantum spin Hall systems using a quantum point contact, Phys. Rev. B 97, 134523 (2018).

[19] A. M. Black-Schaffer and A. V. Balatsky, Odd-frequency superconducting pairing in multiband superconductors, Phys. Rev. B 88, 104514 (2013).

[20] B. Sothmann, S. Weiss, M. Governale, and J. König, Unconventional superconductivity in double quantum dots, Phys. Rev. B 90, 220501(R) (2014).

[21] L. Komendová, A. V. Balatsky, and A. M. Black-Schaffer, Experimentally observable signatures of odd-frequency pairing in multiband superconductors, Phys. Rev. B 92, 094517 (2015).

[22] P. Burset, B. Lu, H. Ebisu, Y. Asano, and Y. Tanaka, Allelectrical generation and control of odd-frequency $s$-wave Cooper pairs in double quantum dots, Phys. Rev. B 93, 201402(R) (2016).

[23] H. Ebisu, B. Lu, J. Klinovaja, and Y. Tanaka, Theory of timereversal topological superconductivity in double Rashba wires: Symmetries of Cooper pairs and Andreev bound states, Prog. Theor. Exp. Phys. 2016, 083 I01 (2016).

[24] M. Eschrig, T. Löfwander, T. Champel, J. C. Cuevas, J. Kopu, and G. Schön, Symmetries of pairing correlations in superconductor-ferromagnet nanostructures, J. Low Temp. Phys. 147, 457 (2007).

[25] C. Triola and A. V. Balatsky, Odd-frequency superconductivity in driven systems, Phys. Rev. B 94, 094518 (2016).

[26] J. Cayao, C. Triola, and A. M. Black-Schaffer, Floquet engineering bulk odd-frequency superconducting pairs, Phys. Rev. B 103, 104505 (2021).

[27] N. Moiseyev, Non-Hermitian Quantum Mechanics (Cambridge University Press, Cambridge, UK, 2011).

[28] K. Kawabata, K. Shiozaki, M. Ueda, and M. Sato, Symmetry and Topology in Non-Hermitian Physics, Phys. Rev. X 9, 041015 (2019).

[29] R. El-Ganainy, K. G. Makris, M. Khajavikhan, Z. H. Musslimani, S. Rotter, and D. N. Christodoulides, NonHermitian physics and PT symmetry, Nat. Phys. 14, 11 (2018).

[30] E. J. Bergholtz, J. C. Budich, and F. K. Kunst, Exceptional topology of non-Hermitian systems, Rev. Mod. Phys. 93, 015005 (2021).

[31] Y. Ashida, Z. Gong, and M. Ueda, Non-Hermitian physics, Adv. Phys. 69, 249 (2020).

[32] T. Kato, Perturbation Theory of Linear Operators (Springer, New York, 1966).

[33] W. Heiss, Exceptional points - their universal occurrence and their physical significance, Czech. J. Phys. 54, 1091 (2004). 
[34] M. V. Berry, Physics of nonhermitian degeneracies, Czech. J. Phys. 54, 1039 (2004).

[35] W. D. Heiss, The physics of exceptional points, J. Phys. A: Math. Theor. 45, 444016 (2012).

[36] C. Dembowski, H.-D. Gräf, H. L. Harney, A. Heine, W. D. Heiss, H. Rehfeld, and A. Richter, Experimental Observation of the Topological Structure of Exceptional Points, Phys. Rev. Lett. 86, 787 (2001).

[37] S.-B. Lee, J. Yang, S. Moon, S.-Y. Lee, J.-B. Shim, S. W. Kim, J.-H. Lee, and K. An, Observation of an Exceptional Point in a Chaotic Optical Microcavity, Phys. Rev. Lett. 103, 134101 (2009).

[38] Y. Choi, S. Kang, S. Lim, W. Kim, J.-R. Kim, J.-H. Lee, and K. An, Quasieigenstate Coalescence in an Atom-Cavity Quantum Composite, Phys. Rev. Lett. 104, 153601 (2010).

[39] T. Gao, E. Estrecho, K. Bliokh, T. Liew, M. Fraser, S. Brodbeck, M. Kamp, C. Schneider, S. Höfling, Y. Yamamoto et al., Observation of non-Hermitian degeneracies in a chaotic exciton-polariton billiard, Nature (London) 526, 554 (2015).

[40] J. Doppler, A. A. Mailybaev, J. Böhm, U. Kuhl, A. Girschik, F. Libisch, T. J. Milburn, P. Rabl, N. Moiseyev, and S. Rotter, Dynamically encircling an exceptional point for asymmetric mode switching, Nature (London) 537, 76 (2016).

[41] L. Feng, Z. J. Wong, R.-M. Ma, Y. Wang, and X. Zhang, Singlemode laser by parity-time symmetry breaking, Science 346, 972 (2014).

[42] B. Peng, Ş. K. Özdemir, M. Liertzer, W. Chen, J. Kramer, H. Yilmaz, J. Wiersig, S. Rotter, and L. Yang, Chiral modes and directional lasing at exceptional points, Proc. Natl. Acad. Sci. USA 113, 6845 (2016).

[43] M. P. Hokmabadi, A. Schumer, D. N. Christodoulides, and M. Khajavikhan, Non-hermitian ring laser gyroscopes with enhanced Sagnac sensitivity, Nature (London) 576, 70 (2019).

[44] M. Parto, Y. G. Liu, B. Bahari, M. Khajavikhan, and D. N. Christodoulides, Non-Hermitian and topological photonics: Optics at an exceptional point, Nanophotonics 10, 403 (2020).

[45] W. Chen, Ş. K. Özdemir, G. Zhao, J. Wiersig, and L. Yang, Exceptional points enhance sensing in an optical microcavity, Nature (London) 548, 192 (2017).

[46] H. Hodaei, A. U. Hassan, S. Wittek, H. Garcia-Gracia, R. El-Ganainy, D. N. Christodoulides, and M. Khajavikhan, Enhanced sensitivity at higher-order exceptional points, Nature (London) 548, 187 (2017).

[47] J. Wiersig, Review of exceptional point-based sensors, Photonics Res. 8, 1457 (2020).

[48] J. C. Budich and E. J. Bergholtz, Non-Hermitian Topological Sensors, Phys. Rev. Lett. 125, 180403 (2020).

[49] S. Bandyopadhyay and A. Balatsky, Classification of superconducting orders in nonhermitian systems, arXiv:2004.02206.

[50] D. Kavický and R. Hlubina, Dynes-like superconductivity in thin al films in parallel magnetic fields, Phys. Rev. B 102, 014508 (2020).

[51] G. D. Mahan, Many-Particle Physics (Springer, Berlin, 2013).

[52] A. Zagoskin, Quantum Theory of Many-Body Systems: Techniques and Applications (Springer, Berlin, 2014).

[53] R. A. Horn and C. R. Johnson, Matrix Analysis, 2nd ed. (Cambridge University Press, Cambridge, U.K., 2013).

[54] Note that, without loss of generality, we consider negative imaginary terms of the eigenvalues but similar conclusions are obtained with positive imaginary terms. Moreover, here we do not consider $\mathcal{P} \mathcal{T}$-symmetric systems which can exhibit real spectra despite being non-Hermitian (see, e.g., Ref. [31]).

[55] D. Pikulin and Y. V. Nazarov, Topological properties of superconducting junctions, JETP Lett. 94, 693 (2012).

[56] D. I. Pikulin and Y. V. Nazarov, Two types of topological transitions in finite Majorana wires, Phys. Rev. B 87, 235421 (2013).

[57] P. San-Jose, J. Cayao, E. Prada, and R. Aguado, Majorana bound states from exceptional points in non-topological superconductors, Sci. Rep. 6, 21427 (2016).

[58] J. Avila, F. Peñaranda, E. Prada, P. San-Jose, and R. Aguado, Non-Hermitian topology as a unifying framework for the Andreev versus Majorana states controversy, Commun. Phys. 2, 133 (2019).

[59] J. Shabani, M. Kjaergaard, H. J. Suominen, Y. Kim, F. Nichele, K. Pakrouski, T. Stankevic, R. M. Lutchyn, P. Krogstrup, R. Feidenhans'l, S. Kraemer, C. Nayak, M. Troyer, C. M. Marcus, and C. J. Palmstrøm, Two-dimensional epitaxial superconductor-semiconductor heterostructures: A platform for topological superconducting networks, Phys. Rev. B 93, 155402 (2016).

[60] M. Kjærgaard, F. Nichele, H. J. Suominen, M. P. Nowak, M. Wimmer, A. R. Akhmerov, J. A. Folk, K. Flensberg, J. Shabani, C. J. Palmstrøm, and C. M. Marcus, Quantized conductance doubling and hard gap in a two-dimensional semiconductor-superconductor heterostructure, Nat. Commun. 7, 12841 (2016).

[61] H. J. Suominen, M. Kjaergaard, A. R. Hamilton, J. Shabani, C. J. Palmstrøm, C. M. Marcus, and F. Nichele, Zero-Energy Modes From Coalescing Andreev States in a Two-Dimensional Semiconductor-Superconductor Hybrid Platform, Phys. Rev. Lett. 119, 176805 (2017).

[62] C. Bøttcher, F. Nichele, M. Kjaergaard, H. Suominen, J. Shabani, C. Palmstrøm, and C. Marcus, Superconducting, insulating and anomalous metallic regimes in a gated twodimensional semiconductor-superconductor array, Nat. Phys. 14, 1138 (2018).

[63] L. Casparis, M. R. Connolly, M. Kjaergaard, N. J. Pearson, A. Kringhøj, T. W. Larsen, F. Kuemmeth, T. Wang, C. Thomas, S. Gronin et al., Superconducting gatemon qubit based on a proximitized two-dimensional electron gas, Nat. Nanotechnol. 13, 915 (2018).

[64] J. O’Connell Yuan, K. S. Wickramasinghe, W. M. Strickland, M. C. Dartiailh, K. Sardashti, M. Hatefipour, and J. Shabani, Epitaxial superconductor-semiconductor two-dimensional systems for superconducting quantum circuits, J. Vac. Sci. Technol. A 39, 033407 (2021).

[65] R. M. Lutchyn, E. P. A. M. Bakkers, L. P. Kouwenhoven, P. Krogstrup, C. M. Marcus, and Y. Oreg, Majorana zero modes in superconductor-semiconductor heterostructures, Nat. Rev. Mater. 3, 52 (2018).

[66] H. Zhang, D. E. Liu, M. Wimmer, and L. P. Kouwenhoven, Next steps of quantum transport in Majorana nanowire devices, Nat. Commun. 10, 5128 (2019).

[67] E. Prada, P. San-Jose, M. W. de Moor, A. Geresdi, E. J. Lee, J. Klinovaja, D. Loss, J. Nygård, R. Aguado, and L. P. Kouwenhoven, From Andreev to Majorana bound states in hybrid superconductor-semiconductor nanowires, Nat. Rev. Phys. 2, 575 (2020). 
[68] As we do not consider in-plane inhomogeneities in the junction, the superconducting order parameter does not have to be determined in a self-consistent calculation, but $\Delta$ represents the effective order parameter in the superconductor, set by material specific parameters before coupling to the lead.

[69] S. Datta, Electronic Transport in Mesoscopic Systems (Cambridge University Press, Cambridge, UK, 1997).

[70] S. Kohler, J. Lehmann, and P. Hänggi, Driven quantum transport on the nanoscale, Phys. Rep. 406, 379 (2005).

[71] D. A. Ryndyk, R. Gutiérrez, B. Song, and G. Cuniberti, Green function techniques in the treatment of quantum transport at the molecular scale, in Energy Transfer Dynamics in Biomaterial Systems, edited by I. Burghardt, V. May, D. A. Micha, and E. R. Bittner (Springer, Berlin, 2009), pp. 213-335.

[72] E. J. Bergholtz and J. C. Budich, Non-Hermitian Weyl physics in topological insulator ferromagnet junctions, Phys. Rev. Research 1, 012003(R) (2019).

[73] See Supplemental Material at http://link.aps.org/supplemental/ 10.1103/PhysRevB.105.094502 for details, which includes Refs. [84-86].

[74] This approximation is valid, e.g., in the wideband limit commonly used in quantum transport [69,71].

[75] B. Pannetier and H. Courtois, Andreev reflection and proximity effect, J. Low Temp. Phys. 118, 599 (2000).

[76] T. M. Klapwijk, Proximity effect from an Andreev perspective, J. Supercond. 17, 593 (2004).

[77] J. Cayao and A. M. Black-Schaffer, Odd-frequency superconducting pairing in junctions with Rashba spin-orbit coupling, Phys. Rev. B 98, 075425 (2018).

[78] While small to moderate values of dissipation, via $\Gamma_{\uparrow, \downarrow}$, induce $\mathrm{NH}$ odd $-\omega$ pairs, we note that very large values can destroy superconductivity, as evident from Eq. (7). For obvious reasons we do not consider this latter regime.
[79] Although the $\operatorname{sgn}(\omega) / \omega^{2}$ behavior of the exceptional odd- $\omega$ pairing has not been found in other systems, odd- $\omega$ pairing acquires an interesting dependence in topological superconductors where it exhibits a $1 / \omega$ behavior around $\omega=0$ in the presence of Majorana states [9].

[80] S. Hüfner, Photoelectron Spectroscopy: Principles and Applications (Springer, Berlin, 2013).

[81] B. Lv, T. Qian, and H. Ding, Angle-resolved photoemission spectroscopy and its application to topological materials, Nat. Rev. Phys. 1, 609 (2019).

[82] T. Yu, C. E. Matt, F. Bisti, X. Wang, T. Schmitt, J. Chang, H. Eisaki, D. Feng, and V. N. Strocov, The relevance of ARPES to high- $T_{c}$ superconductivity in cuprates, npj Quantum Mater. 5, 1 (2020).

[83] With the advent of high-resolution $(\sim 70 \mu \mathrm{eV})$ ARPES, also superconductors with low critical temperatures are accessible $[87,88]$, but alternative probes might also involve transport across the junction, such as conductance measurements [60].

[84] J. Cayao, E. Prada, P. San-Jose, and R. Aguado, SNS junctions in nanowires with spin-orbit coupling: Role of confinement and helicity on the subgap spectrum, Phys. Rev. B 91, 024514 (2015).

[85] A. A. Zyuzin and P. Simon, Disorder-induced exceptional points and nodal lines in Dirac superconductors, Phys. Rev. B 99, 165145 (2019).

[86] B. Michen, T. Micallo, and J. C. Budich, Exceptional nonHermitian phases in disordered quantum wires, Phys. Rev. B 104, 035413 (2021).

[87] T. Shimojima, K. Okazaki, and S. Shin, Low-temperature and high-energy-resolution laser photoemission spectroscopy, J. Phys. Soc. Jpn. 84, 072001 (2015).

[88] J. A. Sobota, Y. He, and Z.-X. Shen, Angle-resolved photoemission studies of quantum materials, Rev. Mod. Phys. 93, 025006 (2021). 